\title{
Efficient Record Linkage in Large Data Sets
}

\author{
Liang Jin, Chen Li, and Sharad Mehrotra \\ Department of Information and Computer Science \\ University of California, Irvine, CA 92697, USA \\ \{liangj,chenli,sharad\}@ics.uci.edu
}

\begin{abstract}
This paper describes an efficient approach to record linkage. Given two lists of records, the record-linkage problem consists of determining all pairs that are similar to each other, where the overall similarity between two records is defined based on domain-specific similarities over individual attributes constituting the record. The record-linkage problem arises naturally in the context of data cleansing that usually precedes data analysis and mining. We explore a novel approach to this problem. For each attribute of records, we first map values to a multidimensional Euclidean space that preserves domain-specific similarity. Many mapping algorithms can be applied, and we use the FastMap approach as an example. Given the merging rule that defines when two records are similar, a set of attributes are chosen along which the merge will proceed. A multidimensional similarity join over the chosen attributes is used to determine similar pairs of records. Our extensive experiments using real data sets show that our solution has very good efficiency and accuracy.
\end{abstract}

\section{Introduction}

The record-linkage problem - identifying and linking duplicate records - arises in the context of data cleansing, which is a necessary pre-step to many database applications. Databases frequently contain approximately duplicate fields and records that refer to the same real-world entity, but are not identical, as illustrated by the following example.

EXAMPLE 1.1 A hospital has a database with thousands of patient records. Every year it receives new patient data from other sources, such as the government or local organizations. It is important for the hospital to link the records in its own database with the data from other sources. However, usually the same information (e.g., name, SSN, address, telephone number) can be represented in different formats. For instance, a patient name can be represented as "Tom Franz" or "Franz, T." or other forms. In addition, there could be typos in the data. The main task here is to link records from different databases in the presence of mismatched information.

With the increasing importance of data linkage in a variety of data-analysis applications, developing effective and efficient techniques for record linkage has emerged as an important problem. It is further evidenced by the emergence of numerous organizations (e.g., Trillium, FirstLogic, Vality, DataFlux) that are developing specialized domainspecific record-linkage and data-cleansing tools.

Three primary challenges arise. First, it is important to determine similarity functions to link two records as duplicates [2, 17]. Such a similarity function consists of two levels. First, similarity metrics need to be defined at the level of each field to determine similarity of different values. Next, field-level similarity metrics need to be combined to determine overall similarity between two records. The second challenge is to provide user-friendly interactive tools for users to specify different transformations, and use the feedback to improve the data quality $[3,5,15]$.

The third challenge is that of scale. Often it is computationally prohibitive to apply a nested-loop approach to use the similarity function(s) to compute the distance between each pair of records. This issue has previously been studied in [7], which proposed an approach that first merges two given lists of records, then sorts records based on lexicographic ordering for each "key" attribute. A fixed-size sliding window is applied, and records within a window are checked to determine if they are duplicates using a merging rule for determining similarity between records. Notice that, in general, records with similar values for a given field might not appear close to each other in lexicographic ordering. The effectiveness of the approach is based on the expectation that if two records are duplicates, they will appear lexicographically close to each other in the sorted list based on at least one key. Even if we choose multiple keys, the approach is still susceptible to deterministic data-entry errors, e.g., the first character of a key attribute is always erroneous. It is also difficult to determine a value of the 
window size that provides a "good" tradeoff between accuracy and performance. In addition, it might not be easy to choose good keys to bring similar records close.

Recently many new techniques have a direct bearing on the record-linkage problem. In particular, techniques to map similarity spaces into similarity/distance-preserving multidimensional Euclidean spaces have been developed. Furthermore, many efficient multidimensional similarity joins have been studied. In this paper, we develop an efficient strategy to record linkage that exploits these advances. We first consider the situation that a record consists of a single attribute. The record-linkage problem then reduces to linking similar strings in two string collections based on a given similarity metric. We propose a two-step solution for duplicate identification. In the first step, we combine the two sets of records, and map them into a high-dimensional Euclidean space. (A similar strategy is used in [14] to map event sequences.) Many mapping techniques can be used. As an example, in this paper we focus on the FastMap algorithm [4] due to its simplicity and efficiency. We develop a linear algorithm called "StringMap" to do the mapping, and it is independent of the distance metric.

In the second step, we find similar object pairs in the Euclidean space whose distance is within a new threshold, which is closely related to the old threshold of string distances. Again, many similarity-join algorithms can be used, and we use the algorithm proposed by Hjaltason and Samet [8] as an example. For each object pair in the result of the second step, we check their corresponding strings to see if their original distance is within the original threshold, and find those similar-record pairs. Our approach has the following advantages. (1) It is "open" to many mapping functions (the first step) and high-dimensional similarityjoin algorithms (the second step). (2) It does not depend on a specific similarity function of strings. (3) Extensive experiments on real large data sets show this approach has very good efficiency and accuracy (greater than 99\%).

We next study mechanisms for record linkage when many attributes are used to determine overall record similarity. Specifically, we consider merging rules expressed as logical expressions over similarity predicates based on individual attributes. Such rules would be generated, for example, if a classifier such as a decision tree was used to train an overall similarity function between records using a labeled training set. Given a merging rule, we choose a set of attributes over which the similarity join is performed (as discussed in the single-attribute case), such that similar pairs can be identified with minimal cost.

This work is conducted in the Flamingo Data-Cleansing Project at UC Irvine. The rest of the paper is organized as follows. Section 2 gives the formulation of the problem. Section 3 presents the first step of our solution, which maps strings to a Euclidean space. Section 4 presents the second step that conducts a similarity join in the high-dimensional space. Section 5 studies how to solve the record-linkage problem if we have a merging rule over multiple attributes. In Section 6 we give the results of our extensive experiments. We conclude in Section 7.

\section{Problem Formulation}

Distance Metrics of Attributes: Consider two relations $R$ and $S$ that share a set of attributes $A_{1}, \ldots, A_{p}$. Each attribute $A_{j}$ has a metric $M_{j}$ that defines the difference a value of $R . A_{j}$ and a value of $S . A_{j}$. There are many ways to define the similarity metric at the attribute level, and domain-specific similarity measures are critical. We take two commonly-used metrics as examples: edit distance and q-gram distance.

Edit distance, a.k.a. Levenshtein distance [13], is a common measure of textual similarity. Formally, given two strings $s_{1}$ and $s_{2}$, their edit distance, denoted $\Delta_{e}\left(s_{1}, s_{2}\right)$, is the minimum number of edit operations (insertions, deletions, and substitutions) of single characters that are needed to transform $s_{1}$ to $s_{2}$. For instance, $\Delta_{e}$ ("Harrison Ford", "Harison Fort") $=2$. It is known that the complexity of computing the edit distance between strings $s_{1}$ and $s_{2}$ is $O\left(\left|s_{1}\right| \times\left|s_{2}\right|\right)$, where $\left|s_{1}\right|$ and $\left|s_{2}\right|$ are the lengths of $s_{1}$ and $s_{2}$, respectively [18].

Given a string $s$ and an integer $q$, the set of $q$-grams of $s$, denoted $G(s)$, is obtained by sliding a window of length $q$ over the characters of string $s$. For instance:

$$
\begin{aligned}
& G \text { (“Harrison Ford") }=\{\text { 'Ha', 'ar', 'rr', 'ri', 'is', 'so', 'on', 'n } \\
& \text { ', 'F', 'Fo', 'or', 'rd' }\} \text {. } \\
& G \text { ('Harison Fort”')=\{'Ha', 'ar', 'ri', 'is', 'so', 'on', 'n ', ' } \\
& \text { F', 'Fo', 'or', 'rt' }\} \text {. }
\end{aligned}
$$

The relative q-gram distance between two strings $s_{1}$ and $s_{2}$, denoted $\Delta_{q}\left(s_{1}, s_{2}\right)$, is defined as:

$$
\Delta_{q}\left(s_{1}, s_{2}\right)=1-\frac{\left|G\left(s_{1}\right) \cap G\left(s_{2}\right)\right|}{\left|G\left(s_{1}\right) \cup G\left(s_{2}\right)\right|}
$$

For example, $\Delta_{q}$ ("Harrison Ford", "Harison Fort") $=1$ $\frac{10}{13} \approx 0.23$. Clearly the smaller the relative q-gram distance between two strings is, the more similar they are.

Similarity Merging Rule: Given distance metrics for attributes $A_{1}, A_{2}, \ldots, A_{p}$, there is an overall function that determines whether or not two records are to be considered as duplicates. Such a function may either be supplied manually by a human, or alternatively, learned automatically using a classification technique. While the specifics of the method used to learn such a function are not of interest to us in this paper, we do make an assumption that the function is captured in the form of a rule discussed below. The form of rules considered include those that could be learned using inductive rule-based techniques such as decision trees. 
Furthermore, this form of merging rules are consistent with the merging functions considered in [7].

Let $r$ and $s$ be two records whose similarity is being determined. A merging rule for records $r$ and $s$ is of the the following disjunctive format.

$$
\begin{array}{rlll} 
& M_{1}\left(A_{1}\right) \leq \delta_{1,1} \wedge & \ldots & \wedge M_{p}\left(A_{p}\right) \leq \delta_{1, p} \\
\vee & M_{1}\left(A_{1}\right) \leq \delta_{2,1} \wedge & \ldots & \wedge M_{p}\left(A_{p}\right) \leq \delta_{2, p} \\
& & & \\
\vee & M_{1}\left(A_{1}\right) \leq \delta_{k, 1} \wedge & \ldots & \wedge M_{p}\left(A_{p}\right) \leq \delta_{k, p}
\end{array}
$$

For each conjunct $M_{j}\left(A_{j}\right) \leq \delta_{i, j}(i=1, \ldots, k$, and $j=$ $1, \ldots, p)$, the value $\delta(i, j)$ is a threshold using the metric function $M_{j}$ on attribute $A_{j}$. The conjunct means that two records $r$ and $s$ should satisfy $M_{j}\left(r . A_{j}, s . A_{j}\right) \leq \delta_{i, j}$.

For instance, given three attributes about papers, title ("T"), name ("N"), and year ("Y"), suppose we use the edit distance $\Delta_{e}$ as a metric for attributes name and year, and the relative q-gram function $\Delta_{q}$ as a metric for attribute title. Then we could have the following rule:

$$
\checkmark \begin{aligned}
\Delta_{q}(T) & \leq 0.10 \wedge \Delta_{e}(N) \leq 4 \wedge \Delta_{e}(Y) \leq 1 \\
\vee \quad \Delta_{q}(T) & \leq 0.15 \wedge \Delta_{e}(N) \leq 2 \wedge \Delta_{e}(Y) \leq 2
\end{aligned}
$$

The record-linkage problem is to find pairs of records $(r, s)$ from relations $R$ and $S$, such that each pair satisfies the merging rule defined above. The quadratic nested-loop solution is not desirable, since as the size of the data set increases, this solution becomes computationally prohibitive. For instance, in our experiments, it took more than 6 hours to use this approach on two single-attribute relations, each with just $10 K$ names, assuming the edit-distance function is used (see Section 6 for our experimental setting). Since by definition, the record-linkage problem is based on approximate matching of those individual metrics for different attributes, an efficient solution that might miss some pairs (but with a high accuracy) can often be more preferable.

\section{Mapping Strings to Euclidean Space}

We first consider the single-attribute case, where $R$ and $S$ share one attribute $A$. Thus the record-linkage problem reduces to linking similar strings in $R$ and $S$ based on a given similarity metric. Formally, given a predefined threshold value $\delta$, we want to find pairs of strings $(r, s)$ from $R$ and $S$ respectively, such that according to the metric $M$ of attribute $A$, the distance of $r$ and $s$ is within $\delta$, i.e.,

$$
M(r . A, s . A) \leq \delta .
$$

Such a string pair is called a similar-string pair; otherwise, it is called a dissimilar-string pair. Our proposed approach has two steps. In the first step, we map strings to objects in a multidimensional Euclidean space, such that the mapped space preserves the original string distances. In the second step, a multi-dimensional similarity join is conducted in the Euclidean space. In this section we discuss the first step.

\subsection{StringMap}

In the first step, we combine the strings from the two relations into one set, and embed them into a Euclidean space. Many mapping/embedding functions can be used, such as multidimensional scaling [12, 19], FastMap [4], Lipschitz [1], SparseMap [10], and MetricMap [16]. These algorithms have different properties in terms of their efficiency, contractiveness, and stress. (See [9] for a good survey.) In this paper we use the FastMap algorithm because of its efficiency and distance-preserving capability. We modify the FastMap slightly and propose an algorithm called "StringMap," as shown in Figure 1 (a). StringMap iterates to find pivot strings to form $d$ orthogonal directions, and computes the coordinates of the $N$ strings on the $d$ axes. The function ChoosePivot (int h, Metric $M$ ) selects two strings to form an axis for the $d$-th dimension. These two strings should be as far from each other as possible, and the function iterates $m$ times to find the seeds. A typical $m$ value could be 5 . The algorithm assumes the dimensionality $d$ of the target space, and we will discuss how to choose a good $d$ value shortly.

Function GetDistance (int a, int b, int $h$, Metric $M)$ computes the distance between strings (indexed by $a$ and $b)$ after they are mapped to the first $h-1$ axes. As shown in Figure 1 (b), it iterates over the $h-1$ dimensions, and does the computation using only the two strings and their already-computed coordinates on the $h-1$ dimensions. There are a few observations about the algorithm. (1) It removes the recursion in FastMap. (2) The computation of coord $[i, h]$ is not symmetric with respect to values $x$ and $y$. (3) In function GetDistance(), it is known that dist $*$ dist $-w * w$ can be negative [16]. In StringMap, we take the square root of the absolute value to compute the new distance. In our experiments we tried other ways to deal with this case, as described in [9]. Our results indicated that the approach of taking the absolute value keeps the distance well.

All the steps in StringMap are linear on the number of strings $N$. Its complexity is $O\left(d^{2} \times m \times N\right)$, assuming it takes $O(1)$ time to compute $M(a, b)$. Notice that a major cost in the algorithm is spent in function ChoosePivot(). We can reduce the cost in the function as follows. At each step in the function, we want to find a new string that is as far from a string (e.g., $t\left[s_{a}\right]$ ) as possible. Instead of scanning the whole $N$ strings, we can just do sampling to find a string that is very far from $t\left[s_{a}\right]$. Or we can just stop once we find a string that is "far enough" from $t\left[s_{a}\right]$, i.e., their distance is above certain value.

\subsection{Choosing Dimensionality $d$}

A good dimensionality value $d$ used in StringMap should have the property that after the mapping, similar strings can 


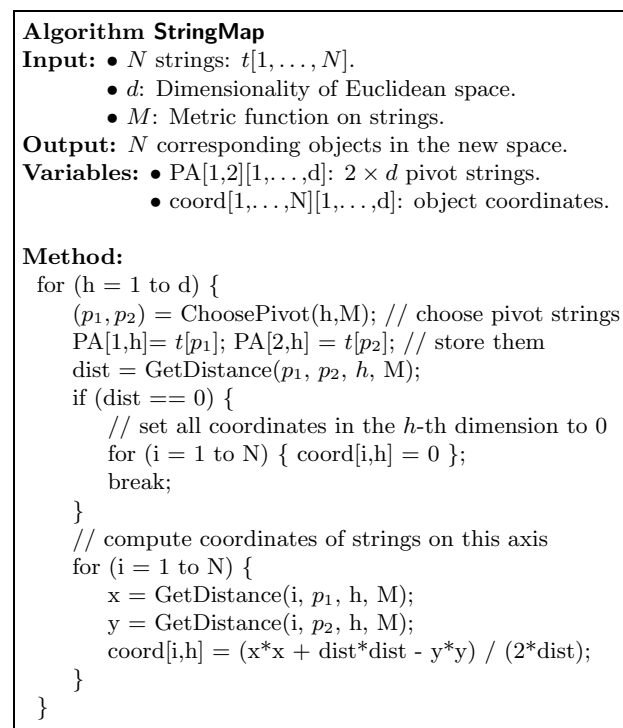

(a) The main algorithm

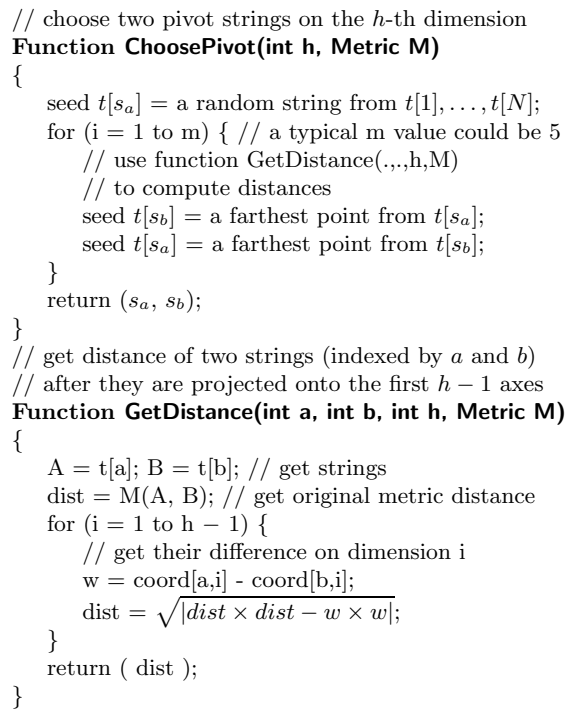

(b) Functions

Figure 1. Algorithm StringMap.

be differentiated from those dissimilar ones. It cannot be too small, since otherwise those dissimilar pairs will not "fall apart" from each other. In particular, the distances of similar-string pairs are too close to those of dissimilar ones. $d$ cannot be too high either, since (1) the complexity of the StringMap algorithm (see above) is linear to $d^{2}$; (2) we need to do a similarity join in the second step, and we want to avoid the curse of dimensionality. As $d$ increases, it becomes more time-consuming to find object pairs whose distance is within a new threshold. We choose a dimensionality $d$ as follows.

1. Randomly select a small number (say, $1 \mathrm{~K}$ ) of strings from the data sets $R$ and $S$. Use the nested-loop approach to find all similar-string pairs within threshold $\delta$ in the selected strings.

2. Run StringMap using different $d$ values. (Typically $d$ is between 5 and 30.) For each $d$, compute the new distances of the similar-string pairs. Find their largest new distance $w$.

3. Find a dimensionality $d$ with a low cost:

$$
\text { cost }=\frac{\# \text { of object pairs within distance } w}{\# \text { of similar-string pairs }}
$$

Intuitively, the cost is the average number of object pairs we need to retrieve in step 2 for each similarstring pair, if $w$ is used as the new threshold $\delta^{\prime}$ for selecting similar-object pairs in the mapped space.
Notice that we only use the pairs of selected strings to compute the cost. This value measures how well a new threshold $\delta^{\prime}=w$ differentiates the similar-string pairs from those dissimilar pairs. In particular, the string pairs whose new distance is within $\delta^{\prime}$ will be retrieved in step 2. Thus the lower the cost is, relatively the fewer object pairs need to be retrieved in step 2 whose original distance is more than $\delta$. Figure 3 in Section 6.1 shows that typically a good dimensionality value is between 15 and 25 .

\section{Similarity Join in Euclidean Space}

In the second step, we find object pairs whose Euclidean distance is within a new threshold $\delta^{\prime}$. For each candidate pair, we check the distance of their original strings to see it is within threshold $\delta$.

\subsection{Choosing New Threshold $\delta^{\prime}$}

The selection of the new threshold $\delta^{\prime}$ depends on the mapping function. For instance, we can set $\delta^{\prime}=\delta$ if the mapping function is contractive. That is, for any two strings $r$ and $s$, we have $M(r, s) \leq M^{\prime}\left(r^{\prime}, s^{\prime}\right)$, where $M(r, s)$ is their distance in the original metric space, and $M^{\prime}\left(r^{\prime}, s^{\prime}\right)$ is the new Euclidean distance of the corresponding objects $r^{\prime}$ and $s^{\prime}$ in the new space. In general, suppose there are two constants $c_{1}, c_{2} \geq 1$, such that for any two strings $r$ and $s$, we have: $\frac{1}{c_{1}} \cdot M(r, s) \leq M^{\prime}\left(r^{\prime}, s^{\prime}\right) \leq c_{2} \cdot M(r, s)$, then we can just set $\delta^{\prime}=c_{2} \cdot \delta$. Properties of different mapping functions are studied in [9]. 
Ideally, the threshold $\delta^{\prime}$ should be set to a maximal value of the new distance between any two similar-string pairs in the original space. Then it will guarantee no false dismissals. However, this maximal value could be either too expensive to find (we do not want to have a nested-loop procedure), or the theoretical upper bound could be too large. Since it is acceptable to miss a few pairs, we would like to choose a threshold such that for most of the similar-string pairs, their new distances are within this threshold. As shown in our experimental results, even though a theoretical upper bound could be large, most of the new distances could be within a much smaller threshold.

In our approach to selecting the dimensionality $d$, the threshold $w$ can be used to identify similar pairs in the mapped space. Here is how we choose the threshold $\delta^{\prime}$. We randomly select a small number (say, $1 \mathrm{~K}$ ) of strings from data sets $R$ and $S$. (Notice these selected strings might be different from those used to decide the dimensionality $d$ in Section 3.2.) We find all the similar-string pairs in these selected strings, and compute their new Euclidean distances after StringMap. We choose their maximal new distance as the new threshold $\delta^{\prime}$. We may do this sampling multiple times (on different sets of selected strings), and choose $\delta^{\prime}$ as the maximal new distance of those similar-string pairs.

\subsection{Finding Object Pairs within $\delta^{\prime}$}

We want to find all those object pairs whose new distance is within this new threshold $\delta^{\prime}$. Many similarity-join algorithms can be applied, and we use a simplified version of the algorithm in [8] as an example, due to its simplicity and availability of the code. We briefly explain the main idea of the algorithm. We first build two R-trees for the mapped objects of the two string sets, respectively. We traverse the two trees from the roots to the leaf nodes to find those pairs of objects within distance $\delta^{\prime}$. As we do the traversal, a queue is used to store pairs of nodes (internal nodes or leaf nodes) from the two trees. ${ }^{1}$ We only insert those node pairs that can potentially yield object pairs that satisfy the condition. Those node pairs that cannot produce results are pruned in the traversal, i.e., they are never inserted into the queue.

Take Figure 2 as an example. Initially, a pair of the root nodes $\left(R_{0}, S_{0}\right)$ is inserted into the queue. At each step, we dequeue the head pair $\left(R_{i}, S_{j}\right)$. If both nodes are internal nodes, we consider all the pairs of their children. For each pair $\left(R_{a}, S_{b}\right)$, we compute their "distance," which is a lower bound of all the distances of their child objects. (The case of a node-object pair is handled similarly.) We prune node pairs as follows: if the distance of a node pair is greater than $\delta^{\prime}$, we do not insert this pair into the queue. If the distance of two nodes is within $\delta^{\prime}$, we insert this pair into the queue.

\footnotetext{
${ }^{1}$ Since we just want to find those object pairs whose distance is within $\delta^{\prime}$, we do not need a priority queue.
}

When we consider the two child nodes of each of the two root nodes in the figure, we have four pairs:

$$
\left(R_{1}, S_{1}\right),\left(R_{1}, S_{2}\right),\left(R_{2}, S_{1}\right),\left(R_{2}, S_{2}\right)
$$

Suppose each pair has a distance within $\delta^{\prime}$, then we insert them into the queue. We remove the pair $\left(R_{1}, S_{1}\right)$ from the queue, and consider pairs of their child nodes:

$$
\left(R_{3}, S_{3}\right),\left(R_{3}, S_{4}\right),\left(R_{3}, S_{5}\right),\left(R_{4}, S_{3}\right),\left(R_{4}, S_{4}\right),\left(R_{4}, S_{5}\right)
$$

For each pair, we compute their distance. If, for example, the distance between $R_{3}$ and $S_{4}$ is greater than $\delta^{\prime}$, we will not consider this pair, since they cannot generate object pairs that have a distance within $\delta^{\prime}$. In other words, all the pairs of their descendants are greater than $\delta^{\prime}$.

Suppose only $\left(R_{3}, S_{3}\right),\left(R_{3}, S_{5}\right),\left(R_{4}, S_{4}\right),\left(R_{4}, S_{5}\right)$ have a distance within $\delta^{\prime}$. Then we insert these pairs into the queue. The status of the queue is shown in the figure. Eventually we have a pair of objects from the queue. Then we compute their Euclidean distance to check if it is within $\delta^{\prime}$. If so, we compute the original metric distance of their original strings. We output this pair of strings if their original metric distance is within the original threshold $\delta$.

Different strategies can be used to traverse the two Rtrees, such as depth first and breadth first. Our experiments show that the depth-first traversal strategy has several advantages. (1) It can effectively reduce the queue size, since object pairs in the leaf nodes can be processed early, and they can be dequeued. Thus the memory requirement of the algorithm tends to be small. In our experiments, when each data set had about $27 \mathrm{~K}$ strings, the breadth-first strategy required about 1.2GB memory, while the depth-first strategy only requested about $30 \mathrm{MB}$ memory. (2) It also reduces the time that the first pair is output, since it can reach the leaf nodes more quickly than the breadth-first strategy.

Comparison: In [6], the authors proposed an attractive q-gram-based approach to find similar string pairs in a DBMS. Compared to that approach, our approach has several advantages in the context of record linkage. First, the distance metrics used at individual attributes might not be edit distance. As argued earlier, domain-specific methods work better in identifying similar records. Furthermore, the algorithm in [6] is geared towards finding all the string pairs that are within a fixed edit-distance threshold. Since the record-linkage problem, by definition, is based on approximate matching, solutions that might miss some such pairs (say those that obtain around $99 \%$ accuracy) but result in significant time savings might be more preferable. As we will see in Section 6.3, our algorithm works very efficiently, while still achieving a very high accuracy. In addition, the implementation of the qgram-based approach inside a database might be less efficient than a direct implementation using other languages (e.g., the $\mathrm{C}$ language). 


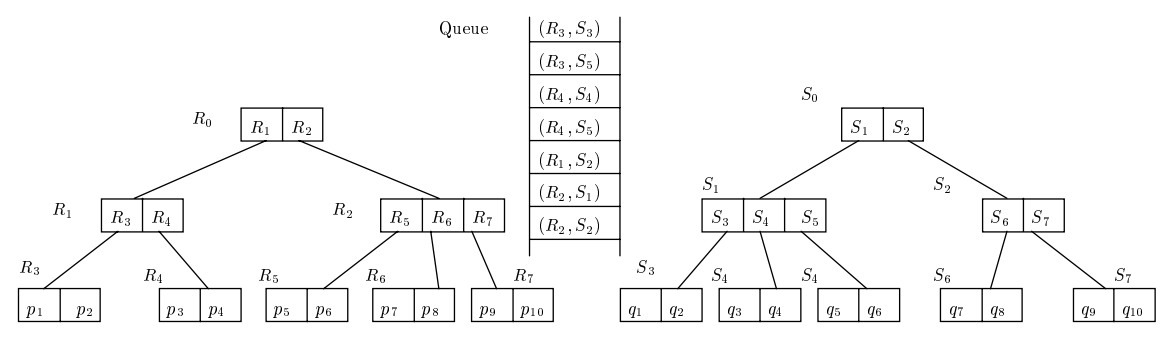

Figure 2. Finding similar-object pairs using R-trees.

\section{Combining Multiple Attributes}

In this section we discuss how to join over multiple attributes efficiently where the merging rule is of the disjunctive normal form (DNF) discussed in Section 2.

Single Conjunctive Clause: For a single conjunctive clause, we can process the most "selective" attribute to find the candidate pairs that satisfy this conjunct condition, and then check other conjunct conditions. For instance, consider the following query clause $Q_{2}$.

$$
\Delta_{q}(\text { title }) \leq 0.15 \wedge \Delta_{e}(\text { name }) \leq 3 \wedge \Delta_{e}(\text { year }) \leq 1
$$

We could first do a similarity search to find all the record pairs that satisfy the first condition, $\Delta_{q}($ title $) \leq 0.15$. For each of the returned candidate pairs, we check if it satisfies the other two conditions on the name and year attributes. Alternatively, we can choose either name or year to do the similarity join. Our experiments show that the step of testing other attributes takes relatively much less time than the step of finding the candidate record pairs, thus we mainly focus on the time of doing the similarity join that finds the candidate pairs. We can use existing techniques on estimating the performance of spatial joins (e.g., [11]), and choose the attribute that takes the least time to do the corresponding similarity join. (This attribute is called the most selective attribute for this conjunctive clause.) Notice that similar to [7], we could also search along multiple attributes of the conjunction to improve accuracy. Since the mapping in Step 1 does not guarantee that all the relevant string pairs will be found, using multiple attributes may improve accuracy. However, as will be shown in the experimental section, since our strategy for single attributes is able to identify matching string pairs at a very high accuracy (over 99\%), traversals along multiple attribute are not necessary. In contrast, since string lexicographic ordering may not preserve domain-specific similarity as well, to get the same degree of accuracy, the approach in [7] requires traversals along multiple keys.

Disjunctive Clauses: The problem becomes more challenging in the case of multiple conjunctive clauses. Take query $Q_{1}$ in Section 2 as an example. We have at least the following different approaches to answering this query.
1. Approach A: Find all record pairs that satisfy the first conjunctive clause by doing a similarity search using the conjunct $\Delta_{q}($ title $) \leq 0.10$. Find all record pairs satisfying the second conjunctive clause by doing a similarity search using the conjunct $\Delta_{e}$ (name) $\leq 2$. Take the union of these two sets of results.

2. Approach B: Do a similarity search to find record pairs that satisfy $\Delta_{q}($ title $) \leq 0.15$ in the second conjunctive clause. These pairs also include all the pairs satisfying the first conjunctive clause, since $\Delta_{q}($ title $) \leq 0.10$ implies $\Delta_{q}($ title $) \leq 0.15$. Among all these pairs, find those satisfying the merging rule.

Approach A needs to do two similarity searches, while approach B requires only one. However, both similarity searches in approach A could be more selective than the single similarity search in approach B. Which approach is better depends on the data set.

The example shows that to answer a conjunctive clause, we can choose at most one conjunct in it to do a similarity join. In addition, once we choose a conjunct $M_{j}\left(A_{j}\right) \leq$ $\delta(i, j)$ to do a similarity join, we do not need to do a similarity join for any other conjunctive clause that has a conjunct $M_{j}\left(A_{j}\right) \leq \delta(k, j)$, where $\delta(k, j) \leq \delta(i, j)$. The reason is that a superset of the results for the conjunct $M_{j}\left(A_{j}\right) \leq$ $\delta(k, j)$ has been returned by the similarity search. As the number of attributes and the number of conjunctive clauses increase, there could be an exponential number of possible ways to answer the query. In fact, assuming the time of doing a similarity search for each conjunct is given, we can show that the problem of finding an optimal solution (with a minimal total time) to answer the query is NP-complete. (The NP-hardness can be proven by reducing the problem to the NP-complete set-cover problem.)

Since it could be too time-consuming to find an optimal solution, we propose two heuristic-based algorithms for finding a good one.

- Algorithm 1: Treat all the conjunctive clauses separately. For each of them, choose the most selective attribute to do the corresponding similarity join. If we choose the same attribute $A_{j}$ in two conjunctive clauses, and their corresponding thresholds $\delta_{i, j} \leq$ 
$\delta_{k, j}$, then we only choose the threshold $\delta_{k, j}$ to do the similarity search for the second clause, saving one similarity search for the first one. Take the union of results of all conjunctive clauses.

- Algorithm 2: For each attribute, choose the largest threshold among all its conjuncts. Among the largest thresholds of different attributes, choose the most selective one to do a similarity join. Among the results, find the record pairs that satisfy the merging rule.

For instance, consider the query $Q_{1}$ in Section 2. Suppose Algorithm 1 chooses $\Delta_{q}($ title $) \leq 0.10$ as the most selective condition for the first clause, and $\Delta_{e}$ (name) $\leq 2$ for the second one. Thus it will produce the approach A above. For Algorithm 2, the largest thresholds of the three attributes title, name, and year are $0.15,4$, and 2, respectively. Suppose $\Delta_{q}($ title $) \leq 0.15$ is the most selective one. This algorithm will produce approach B as the solution. In general, Algorithm 1 works better than Algorithm 2 if doing multiple similarity searches with small thresholds is more efficient than one with a large threshold.

\section{Experiments}

In this section we present our extensive experimental results to evaluate our solution. The following are three main sources we used. (1) Source 1 consists of 54,000 movie star names collected from The Internet Movie Database. ${ }^{2}$ The length of each name varies from 5 to 20 , and their average length is about 12. (2) Source 2 is from the Die Vorfahren Database of mostly Pomeranian surnames and geographic locations. ${ }^{3}$ It contains 133,101 full names, whose lengths are less than 40, and mean length is around 15. (3) Source 3 is from the publications in DBLP. ${ }^{4}$ We randomly selected 20,000 papers in proceedings. We use this data source to show how to do data linkage in multiple-attribute cases.

All the experiments were run on a $\mathrm{PC}$, with a $1.5 \mathrm{GHz}$ Athlon CPU and 512MB memory. The operating system is Windows 2000, and the complier is gnu $\mathrm{C}++$ running in cygwin. This setting shows that our approach does not have specific hardware and software requirements. We used 8, 192 as the page size to build R-trees. Most of our experimental results are similar for three sources. Due to space limitation, we mainly report the results on data source 1 . In addition, since the results for the edit-distance metric and the q-gram distance metric are similar, we will mainly report the results of the edit-distance metric.

\footnotetext{
${ }^{2}$ http: //www.imdb.com/

${ }^{3}$ http://feefhs.org/dpl/dv/indexdv.html

${ }^{4}$ http://www.informatik.uni-trier.de/ ley/db/
}

\subsection{Choosing Dimensionality $d$}

As discussed in Section 3.2, we need to choose a good dimensionality $d$ for the StringMap algorithm. To select a $d$ value, we randomly selected $2 \mathrm{~K}$ strings from Source 1 to form two data sets with the same size, and ran StringMap using different dimensionalities. For edit distance, we considered the case where $\delta=1,2$, and 3 . For the q-gram metric, we considered $\delta=0.1$ and 0.15 .

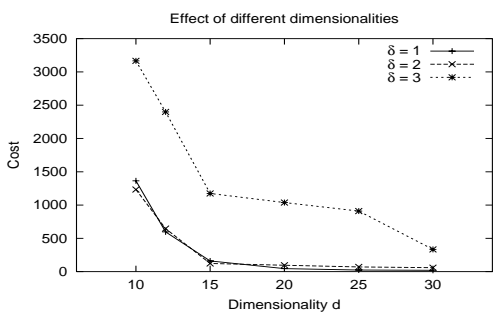

(a) Edit Distance

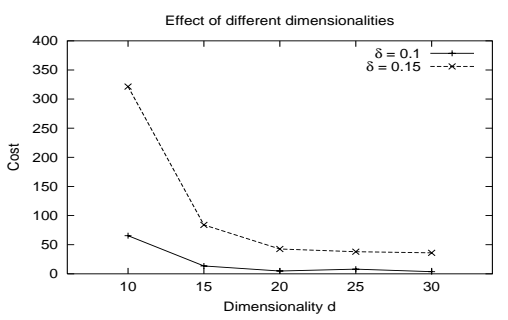

(b) Q-gram Distance

Figure 3. Costs of different dimensionalities.

Figures 3 (a) and (b) show costs for different dimensionalities for edit distance and q-gram distance, respectively. (See Section 3.2 for the definition of "cost.") It is shown that the cost decreased with the increase of dimensionality. That is, the larger the dimensionality, the fewer extra object pairs we need to retrieve in step 2 for each similar-string pair, while the original strings of these objects have a distance greater than $\delta$. On the other hand, due to the computational complexity of StringMap and the curse of dimensionality, $d$ cannot be high either. The results show that $d=20$ is a good dimensionality for both metrics.

Figures 4 (a) and (b) show the distributions of the objectpair distances after StringMap, for the edit-distance metric. We randomly sampled $2 \mathrm{~K}$ from Source 1 to form two data sets with the same size. We chose $\delta=2$ for similarstring pairs, and set $d=20$. The figures show that after StringMap, there is a new threshold to differentiate similar pairs from most dissimilar pairs. In particular, all the sampled similar-string pairs had new object-pair distances within 5.5, while most of dissimilar-string pairs had their 


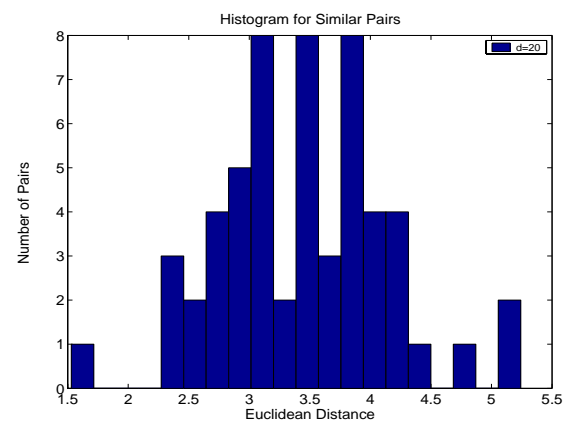

(a) Similar pairs

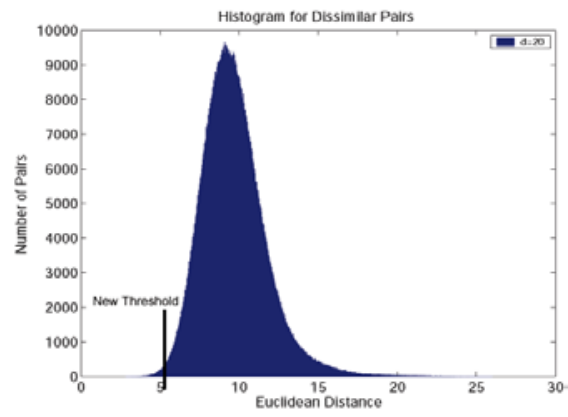

(b) Dissimilar pairs

Figure 4. Histograms of new Euclidean distances: edit distance, $\delta=2, d=20$.

object-pair distances larger than 5.5.

\subsection{Choosing Threshold $\delta^{\prime}$}

As discussed in Section 4.1, we selected new threshold $\delta^{\prime}$ for the second step as follows. For each source, we randomly selected $2 \mathrm{~K}$ strings, and partitioned the strings into two data sets equally. We used the nested-loop approach to find all the similar-string pairs in the selected strings. We ran StringMap with $d=20$, and traced the new Euclidean distances of these sample similar-string pairs. We did this sampling 10 times, and chose $\delta^{\prime}$ as the largest new objectpair distance of the sampled similar-string pairs.

\begin{tabular}{l|c|c|c|c}
\hline & $\Delta_{e}=1$ & $\Delta_{e}=2$ & $\Delta_{e}=3$ & $\Delta_{q}=0.2$ \\
\hline Source 1 & 4.5 & 5.9 & 7.735 & 0.2 \\
Source 2 & 5.36 & 7.0 & 10 & 0.2 \\
\hline
\end{tabular}

Table 1. Threshold $\delta^{\prime}$ used in step $2(d=20)$.

Table 1 shows the $\delta^{\prime}$ values used in step 2 for two different metrics. Notice that when using the edit-distance met- ric, since the two sources have different strings with different length distributions, it is not surprising that we need to choose different $\delta^{\prime}$ values by sampling. For the q-gram metric, we set $\delta^{\prime}=0.2$ for both data sources 1 and 2 .

\subsection{Running Time}

In order to measure the performance of our approach, we ran our algorithm on different data sizes. In each case, we chose the same number of strings in both data sets. We chose dimensionality $d=20$, and let the total size of strings vary from $2 \mathrm{~K}, 4 \mathrm{~K}, 8 \mathrm{~K}, 16 \mathrm{~K}$, to $54 \mathrm{~K}$ from Source 1 . We measured the corresponding running time.

Figure 5 shows the time of the complete algorithm (including two steps), and the time of the StringMap step, assuming we use the edit-distance metric. Their gap is the time of the second step that did the R-tree similarity join. The figure shows that as the data sizes increased, both the StringMap time and the total time grew. Our approach is shown to be very efficient and scalable. For instance, when the total number of strings is $54 K$, it took the approach only 41 minutes to find the similar-string pairs. (It look almost one week for the nested-loop approach to finish.) The figures also indicate that other similarity-join techniques may be used in the second step to improve its performance.

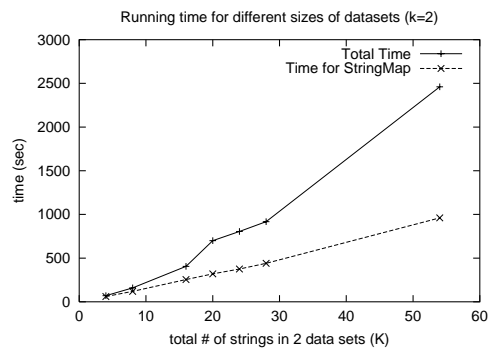

Figure 5. Running time ( $\delta=2, d=20)$, edit distance.

In the case where the edit-distance metric is used, the approach in [6] can be used to find all the string pairs whose edit distance is within a given threshold. We implemented this approach using Oracle 8.1.7 on the same PC, and let the database use indexes to run the query. We selected subsets of strings from Source 1, and let both sets have the same number of strings. In our approach we chose threshold $\delta=2$, dimensionality $d=20$, and new threshold $\delta^{\prime}=5.9$. Figure 6 shows the performance difference between these two approaches. The figure shows that our approach can substantially reduce the time of finding similar-string pairs. Notice that even though our approach cannot guarantee to find all such pairs, as we will see shortly, it can still achieve a very high accuracy. In the context of record linkage, an efficient approximate-search algorithm with a very high accuracy might be more desirable. 


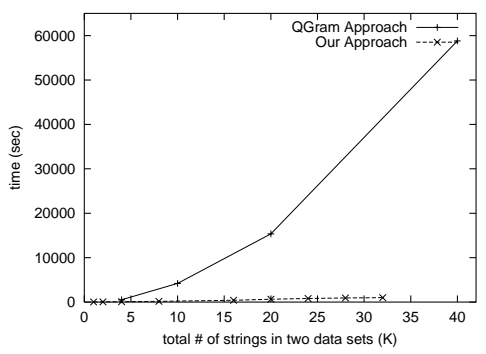

Figure 6. Our approach (approximate search) versus the qgram exact approach in [6].

\subsection{Accuracy}

We want to know how well our approach can find all the similar-string pairs. (Ideally we want to find all of them!) In particular, we are interested in the accuracy, i.e., ratio of similar-string pairs found among all similar-string pairs. Figure 7 shows the accuracy of data source 1, with different threshold $\delta^{\prime}$ values in the second step, using the editdistance metric. As the $\delta^{\prime}$ value increased, the accuracy also increased, and it quickly got very close to $100 \%$. For instance, in the case where $\delta=2$, the accuracy reached $99 \%$ when $\delta^{\prime}=5.6$. When we further increased the threshold, the accuracy continued to grow close to $100 \%$. We also implemented the sliding-window approach in [7]. Without loss of generality, we used attributes as keys, and the condition is $\Delta_{e}$ (name) $\leq 2$ for data source 1 . We chose different window sizes, and for each of them, the time and accuracy were measured.

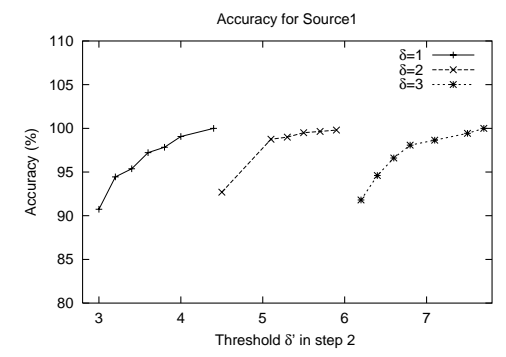

Figure 7. Accuracy versus threshold $\delta^{\prime}(\mathbf{d}=20)$, edit distance.

Figure 8 shows the accuracy and time for these two approaches. It shows that our approach can achieve a very high accuracy given a time limit. The primary reason is that our mapping function provides very good distance/similarity preservation. Since lexicographic ordering does not preserve edit distances as well, the approach discussed in [7] needs to consider a very large window size (and hence cost) to obtain competitive degree of accuracy.

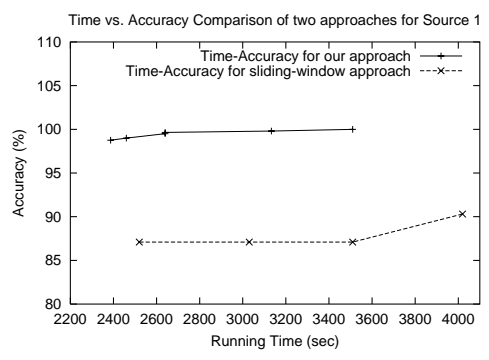

\section{Figure 8. Our approach versus the sliding- window approach in [7].}

\subsection{Results on Multiple Attributes}

Now we report our experimental results for the multipleattribute case on data source 3 .

Single Conjunctive Clause: We evaluated the single conjunctive query $Q_{2}$ in Section 5 . For the three attributes to perform a similarity join: title, name, and year, experimental results showed that the attribute year is not a good one to do a similarity join, since too many candidate pairs were generated. Thus we answered the query by conducting a similarity join using attributes title and name, respectively.

\begin{tabular}{c|c|c}
\hline Similarity-join Attribute & Time $(\mathrm{sec})$ & Final Result Size \\
\hline name & 1140 & 700 \\
title & 1540 & 702 \\
\hline
\end{tabular}

Table 2. Results of similarity join using different attributes.

As shown in Table 2, for the thresholds in the query, doing a similarity join on name is more efficient than on title. Notice that the result size is different for these two similarity searches, since both of them are approximate. The small difference (only two pairs) between them again shows that our approach has a very high accuracy.

Disjunctive Clause: We used the query $Q_{1}$ in Section 2 as an example of disjunctive clauses. We implemented the two approaches in Section 5. For algorithm 1 that produces approach A, we chose the conjunct $\Delta_{q}($ title $) \leq 0.10$ to do the similarity search for the first clause, and conjunct $\Delta_{e}$ (name) $\leq 2$ for the second one. After getting the result pairs for each clause, we took a union of the two sets and produced the final result. Table 3 shows the results. The total time for approach A was 1,820 seconds, and the size of the final result set was 619 . Notice that the results of the two clauses had overlapped record pairs. The accuracy of taking this approach is more than $99 \%$.

For algorithm 2 that produces approach B, we found that $\Delta_{q}($ title $) \leq 0.15$ was the most selective conjunct, using which we performed the similarity join. Table 4 shows the 


\begin{tabular}{c|c|c}
\hline Selected Condition & Time $(\mathrm{sec})$ & \# of Record Pairs \\
\hline Clause $1, \Delta_{q}($ title $) \leq 0.1$ & 1060 & 406 \\
Clause $2, \Delta_{e}($ name $) \leq 2$ & 760 & 518 \\
\hline Total & 1820 & 619 (pairs overlap) \\
\hline
\end{tabular}

Table 3. Approach A for disjunctive clause.

results. In particular, the total time for approach B was 1,743 seconds, and the size of the final result was also 619 .

\begin{tabular}{c|c|c}
\hline Selected Condition & Time & \# of Record Pairs \\
\hline Clause $2, \Delta_{q}($ title $) \leq 0.15$ & 1743 secs & 619 \\
\hline
\end{tabular}

\section{Table 4. Approach B for disjunctive clause.}

For this example, approach B was better than approach A. In general, algorithm 1 produces a solution that tries to minimize the time of each individual similarity join, which tends to produce a small candidate set. Algorithm 2 produces a solution that needs to perform a similarity join only once for all the clauses. It may need more time for the single similarity join, since the threshold could be large. To fix this problem of Algorithm 2, we can set an upper bound on the threshold of each attribute, and we will never consider those conjuncts on this attribute whose threshold is above this bound. One observation is that if the thresholds for the attributes vary a lot, it could be better to take Algorithm 1 . Otherwise, Algorithm 2 could be preferable.

\section{Conclusion}

In this paper we developed a novel approach to the record-linkage problem: given two lists of records, we want to find similar record pairs, where the overall similarity between two records is defined based on domainspecific similarities over individual attributes. For each attribute of records, we first map records to a multidimensional Euclidean space that preserves domain-specific similarity. Given the merging rule that defines when two records are similar, a set of attributes are chosen along which the merge will proceed. A multidimensional similarity join over the chosen attributes is used to determine similar pairs of records. Our extensive experiments using real data sets showed that our solution has very good efficiency and accuracy. In addition, our approach is very extendable, since many existing techniques can be used. It can also be generalized to other similarity functions between strings.

Acknowledgments: We thank Michael OrtegaBinderberger for providing his implementation of the algorithm in [8]. We thank the authors of [6] for providing the details of their implementation. We thank Heikki Mannila for letting us know the work in [14].

\section{References}

[1] J. Bourgain. On Lipschitz embedding of finite metric spaces in hilbert space. Israel Journal of Mathematics, 52(1-2):4652,1985 .

[2] W. W. Cohen, H. A. Kautz, and D. A. McAllester. Hardening soft information sources. In Knowledge Discovery and Data Mining, pages 255-259, 2000.

[3] M. G. Elfeky, V. S. Verykios, and A. K. Elmagarmid. Tailor: A record linkage toolbox. In $I C D E, 2002$.

[4] C. Faloutsos and K.-I. Lin. Fastmap: A fast algorithm for indexing, data-mining and visualization of traditional and multimedia datasets. In M. J. Carey and D. A. Schneider, editors, SIGMOD, pages 163-174, 1995.

[5] H. Galhardas, D. Florescu, D. Shasha, E. Simon, and C.A. Saita. Declarative data cleaning: Language, model, and algorithms. In $V L D B$, pages 371-380, 2001.

[6] L. Gravano, P. G. Ipeirotis, H. V. Jagadish, N. Koudas, S. Muthukrishnan, and D. Srivastava. Approximate string joins in a database (almost) for free. In $V L D B$, pages $491-$ 500, 2001.

[7] M. A. Hernández and S. J. Stolfo. The merge/purge problem for large databases. In M. J. Carey and D. A. Schneider, editors, SIGMOD, pages 127-138, 1995.

[8] G. R. Hjaltason and H. Samet. Incremental distance join algorithms for spatial databases. In L. M. Haas and A. Tiwary, editors, SIGMOD, pages 237-248, 1998.

[9] G. R. Hjaltason and H. Samet. Contractive embedding methods for similarity searching in metric spaces. Technical report, University of Maryland Computer Science, 2000.

[10] G. Hristescu and M. Farach-Colton. Cluster-preserving embedding of proteins. Technical Report 99-50, Rutgers Univ., 81999.

[11] Y.-W. Huang, N. Jing, and E. A. Rundensteiner. A cost model for estimating the performance of spatial joins using R-trees. In Statistical and Scientific Database Management, pages 30-38, 1997.

[12] J. B. Kruskal and M. Wish. Multidimensional Scaling. Sage Piblications, Beverly Hills, CA, 1978.

[13] V. I. Levenshtein. Binary codes capable of correcting deletions, insertions and reversals. Soviet Physics Doklady, 10:707-710, 1966.

[14] H. Mannila and J. K. Seppnen. Recognizing similar situations from event sequences. In First SIAM Conference on Data Mining, 2001.

[15] V. Raman and J. M. Hellerstein. Potter's wheel: An interactive data cleaning system. In The VLDB Journal, pages 381-390, 2001.

[16] J. T.-L. Wang et al. Evaluating a class of distance-mapping algorithms for data mining and clustering. In Knowledge Discovery and Data Mining, pages 307-311, 1999.

[17] W. Winkler. Advanced methods for record linkage, 1994.

[18] P. N. Yianilos and K. G. Kanzelberger. The LIKEIT intelligent string comparison facility. Technical report, NEC Research Institute, 1997.

[19] F. W. Young and R. M. Hamer. Multidimensional Scaling: History. Theory and Applications Erlbaum, New York, 1987. 